\title{
Ionic-Liquid Matrices for Improved Analysis of Phospholipids by MALDI-TOF Mass Spectrometry
}

\author{
Ying L. Li and Michael L. Gross \\ Department of Chemistry, Washington University, St. Louis, Missouri, USA \\ Fong-Fu Hsu \\ Department of Internal Medicine, Washington University School of Medicine, St. Louis, Missouri, USA
}

\begin{abstract}
The use of ionic liquid matrices (ILMs) for phospholipids (PLs) affords higher signal intensity, smaller spot size, improved spot homogeneity, better signal reproducibility, and comparable or better detection limits compared to that of the crystalline matrix 2,5-dihydroxybenzoic acid (2,5-DHB). The ionization products are comparable to those with 2,5-DHB although the use of ILMs gives a stronger tendency to produce alkali-metal-ion adducts and a lower extent of prompt fragmentation. (J Am Soc Mass Spectrom 2005, 16, 679-682) (c) 2005 American Society for Mass Spectrometry
\end{abstract}

$\mathrm{D}$ evelopments in mass spectrometry [1] beginning with fast atom bombardment $[2,3]$ and continuing with electrospray ionization (ESI), have facilitated the structure elucidation of lipids. ESI increases the sensitivity by orders of magnitude and permits interface with liquid chromatography (LC) [4, 5]. Tandem mass spectrometry (MS/MS) provides the fragmentation to obtain structure [6, 7].

Although attempts to use MALDI for PLs were reported as early as $1995[8,9]$, ESI has been the favored ionization method, in part because MALDI does not interface well with LC, and its matrix peaks interfere with low-mass lipid signals [1]. Nevertheless, the tolerance to salt and other contaminates and the easy sample preparation make MALDI an opportunity for lipid analysis [10]. Collisional activation in FT mass spectrometry [9] and prompt fragmentation with time-offlight also [11] make up for some disadvantages.

Most MALDI analyses of PLs have used 2,5-dihydroxybenzoic acid (2,5-DHB) as the matrix because its use causes little fragmentation [8]. Although methanol and acetone that dissolve the matrix also solubilize PLs, their use causes spreading of the sample spots, increasing the difficulty of locating a "sweet spot" [10,12]. Another issue is 2,5-DHB, like all "crystalline matrices," cocrystallizes with the analyte, producing a heterogeneous sample in contrast to ionic liquid matrices (ILMs), which may also be crystalline solids at room temperature. Their crystal size,

Published online March 10, 2005

Address reprint requests to Dr. M. L. Gross, Department of Chemistry, Washington University, One Brookings Drive, St. Louis, MO 63130, USA. E-mail: mgross@wustl.edu however, is smaller, still affording homogeneous samples and better shot-to-shot, spot-to-spot reproducibility.

Our laboratory, in collaboration with Armstrong's [13], introduced ILMs for MALDI and demonstrated their utility for analysis of peptides, proteins, and synthetic polymers. ILMs have strong UV absorbance, low volatility, form homogeneous solutions with analytes, and promote ionization. The homogeneous solutions that result from their use are a key advantage because the search for a "sweet spot" is facilitated and reproducibility improved.

Armstrong and coworkers [14] implemented ILMs for DNA oligomers, and we recently demonstrated significant advantages for quantification of oligonucleotides and peptides [15]. Recently, Stahl et al. [16] evaluated the ILM, butylammonium 2,5-dihydroxybenzoate (DHBB), as a MALDI matrix and demonstrated improved reproducibility, reduced fragmentation, and utility as a cosolvent for conducting enzymatic reactions. Their work established applications of ILMs for analysis of oligosaccharides, glycoconjugates, as well as peptides, proteins, and synthetic polymers [13].

We wish to show for the first time the applicability of ILMs for phospholipids (PL) and the advantages of the small spot sizes and homogeneity of lipid-ILM mixtures over "crystalline matrices."

\section{Experimental}

\section{Materials and Sample Preparation}

$\alpha$-Cyano-4-hydroxycinnamic acid (CHCA), 1-methylimidazole, aniline, pyridine, tripropylamine, tributylamine, and trifluoroacetic acid (TFA) were from Aldrich (Milwaukee, WI). Phospholipids were from 
Table 1. Comparison of 2,5-DHB vs. ILMs of CHCA used as matrices for MALDI-MS of six classes of PLs: PC, PE, PS, PA, PG and $\mathrm{PI}^{\mathrm{a}}$ in both positive (upper row) and negative (lower row)-ion modes. Signal intensities are divided by 1000

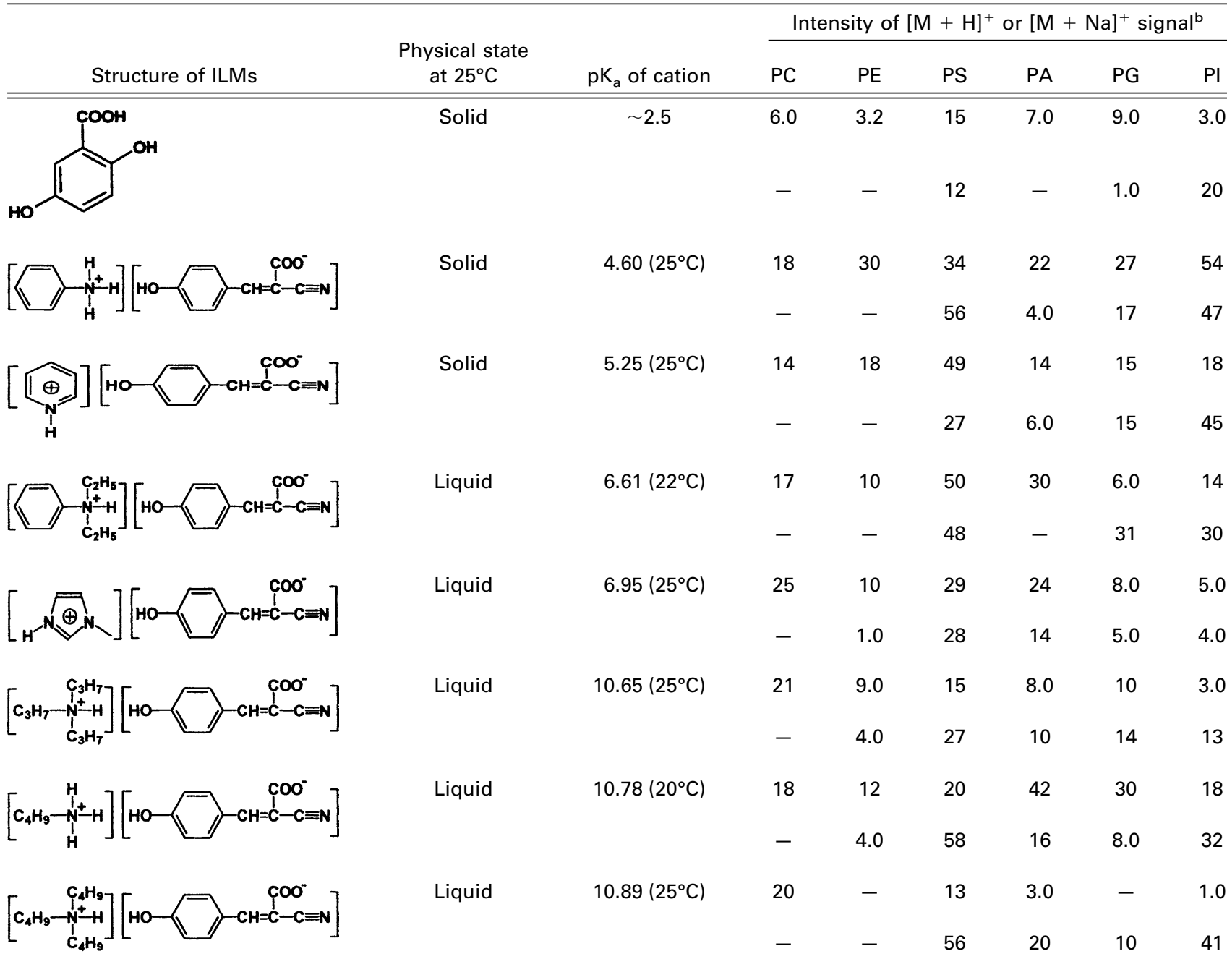

aPhosphatidylcholine ( $P C, 16: 0 / 18: 1$, monoisotopic $M W=759.58)$; phosphatidylethanolamine ( $P E, 16: 0 / 18: 1$, monoisotopic $M W=717.53$ ) phosphatidylserine (PS, 16:0/18:1, monoisotopic MW = 761.52); phosphatidic acid (PA, 16:0/18:1, monoisotopic MW = 674.45); phosphatidylglycerol (PG, 16:0/18:1, monoisotopic MW = 748.53); phosphatidylinositol ( $\mathrm{Pl}$, bovine liver, monoisotopic MW for main product, 18:0/20:4 = 885.55). All measurements are from spotting lipid solutions at concentration of $1 \mu \mathrm{g} / \mu \mathrm{L}$.

${ }^{\text {b}}$ The first row for each phospholipid species indicated signal intensity detected in the positive-ion mode, the second row indicated signal intensity in the negative-ion mode. When no signal was detected, the space is left blank $(-)$.

Avanti Polar Lipids, Inc. (Alabaster, AL). HPLC grade organic solvents were from Fisher (St. Louis, MO). All were of highest available purity and used without further purification. RTILs of CHCA were synthesized as previously reported [13].

Phosphatidylcholine (PC), phosphatidic acid (PA), phophatidylethanolamine (PE), serine (PS), glycerol (PG), and inositol (PI) were dissolved in methanol/chloroform $(50 / 50)$ at $1 \mu \mathrm{g} / \mu \mathrm{L}$ and mixed with the same volume of various ILM solutions, which were prepared as previously reported [13], before spotting on the sample plate.

\section{Instrumentation}

A Voyager DE-STR mass spectrometer (PerSeptive Biosystems, Framingham, MA) equipped with a nitrogen laser (337-nm, 3-ns pulse) was operated in positive- and negativeion reflector modes, using an acceleration of $20 \mathrm{kV}$, grid and guide-wire voltages of $70 \%$ and $0.05 \%$ of the accelerating voltage, and a delay time of $400 \mathrm{~ns}$. The low-mass gate was at $m / z 400$ to filter low-mass matrix ions. Each mass spectrum was the average of 200 laser shots.

\section{Results and Discussion}

\section{Signal Improvement: ILMs versus 2,5-DHB}

For the PL classes, we found improved signals, good solubilizing properties, and longevity in vacuum when using ILMs compared to using 2,5-DHB, the typical "crystalline" matrix for MALDI analysis of PLs $[10,17]$. The ILM contained the needed chro- 



Figure 1. MALDI mass spectra of PG $(16: 0 / 18: 1,1 \mu \mathrm{g} / \mu \mathrm{L})$ in a matrix of (a) 2,5-DHB, (b) ILM butyl ammonium $\alpha$-cyano-4hydroxycinnamate in the positive-ion mode. The structure of ILM is given in Table 1.

mophore and an acidic proton for ionization. The use of various ILMs, however, gave unpredictable MS responses for the analytes (results summarized in Table 1). We have yet to find a consistent relationship between the MS response and any characteristic of the ILMs (e.g., its gas-phase acidity, proton affinity, physical state). Elucidating why subtle changes in the cation component affects ionization is beyond the scope of this short note.

Figures 1 and 2 show two examples of the improvement of the signal for PG (16:0/18:1) when using the ILMs, butyl ammonium and 1-methyl imidazolium $\alpha$-cyano-4-hydroxycinnamate in the positive and negative-ion modes, respectively. From comparison of Figure 1a and Figure 1b, we see three times higher signal intensity for PG in the positive-ion mode at comparable analyte amount and intensity than for the solid matrix. We found similar results in the negative-ion mode (Figure 2a versus Figure 2b).

The use of ILMs gives improved sample homogeneity and signal reproducibility compared to that of the "crystalline matrix" 2,5-DHB. Acquisition of spectra when using 2,5-DHB (Figures 1a and 2a) required searching for a "sweet spot," which was made more difficult by the large spot sizes when 2,5-DHB was introduced in methanol or acetone [10,12]. On the other hand, the use of ILM solutions produced smaller spot sizes than those of 2,5-DHB (2 versus $6 \mathrm{~mm}$ diameter), leading to a more homogeneous response for PLs. For Figures $1 b$ and $2 b$, the spectra were of 200 laser shots on randomly selected positions, and each shot gave a nearly identical mass spectrum, making the experiment amenable to automation.
The detection limits for PLs when using ILMs as matrices are comparable or better than those achieved with 2,5-DHB. To permit comparison, six classes of PLs, in chloroform/methanol $(50 / 50)$ at $1 \mu \mathrm{g} / \mu \mathrm{L}$ were mixed either with 2,5-DHB or an ILM and then analyzed by MALDI. The solutions were then submitted to repeated 10-fold dilutions until the signal became undetectable for both preparations placed on the same sample plate and analyzed at the same time. Detection limits for the PLs PS, PA, PG, and PE in ILMs are comparable to those achieved with 2,5-DHB $(\sim 100$ $\mathrm{ng} / \mu \mathrm{L}$ for PS, PA, and PG, and $\sim 1 \mu \mathrm{g} / \mu \mathrm{L}$ for PE), whereas those for PC and PI are approximately one order of magnitude lower with the ILMs $(\sim 10 \mathrm{ng} / \mu \mathrm{L})$. The reduced fragmentation afforded by ILMs for other analytes [13, 16] also applies for PLs.

Another beneficial aspect for ILMs is the maintenance of relatively neutral $\mathrm{pH}$ when mixed with the analyte solutions. The "crystalline matrices", 2,5-DHB $\left(\mathrm{pK}_{\mathrm{a}} \sim 3.0\right)$ or CHCA $\left(\mathrm{pK}_{\mathrm{a}} \sim 1.2\right)$, are acids, leading to low $\mathrm{pH}$ spotting solutions. This may induce acidic denaturation or hydrolysis of acid-labile molecules. In contrast, ILMs are salts and weaker acids $\left(\mathrm{pK}_{\mathrm{a}}\right.$ values of their cation components are 4.6-11.0). One can choose an ILM that keeps the $\mathrm{pH}$ close to 7 , minimizing problems with acid or base lability.

\section{Ionization Patterns}

PC, PE, PA, PS, PG, and PI in both the positive- and negative-ion modes give molecular ions except PC, which produces signals only in the positive-ion mode.

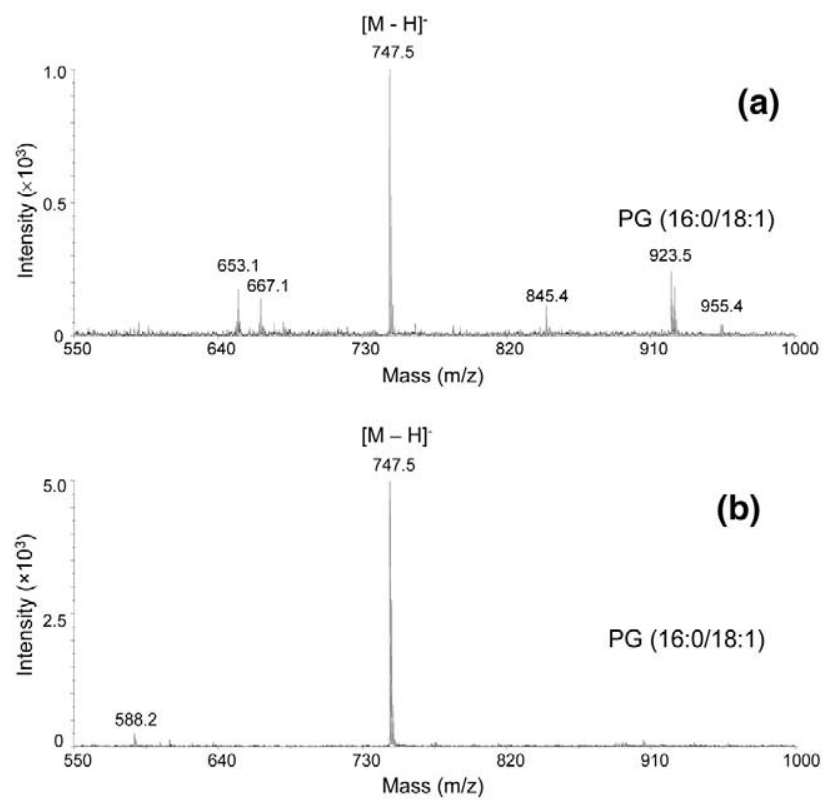

Figure 2. MALDI mass spectra of PG $(16: 0 / 18: 1,1 \mu \mathrm{g} / \mu \mathrm{L})$ in a matrix of (a) 2,5-DHB, (b) ILM 1-methyl imidazolium $\alpha$-cyano-4hydroxycinnamate in the negative-ion mode. The structure of ILM is given in Table 1. The unidentified peaks are probably from unknown impurities. 
Further, analysis of PC gives the lowest detection limit of these classes of PLs. Petković et al. [12] previously showed that PC interfered with the detection of other PLs in the positive-ion mode. The charged quaternary ammonium group of $\mathrm{PC}$ removes the requirement to accept positive ions $\left(\mathrm{H}^{+}\right.$or $\left.\mathrm{Na}^{+}\right)$to cause ionization, making its sensitivity the highest of the six classes of phospholipids. Detecting PC as a negative ion is nearly impossible because a neutral PC is already deprotonated at the phosphate group to compensate for the positive charge of the quaternary ammonium, and further deprotonation is difficult because there are no other acidic groups in the molecule. PE and the other lipid classes, however, can be deprotonated to give negative ions, and indeed, they are observed, albeit weakly for PE.

The PC class of PLs is zwitter-ionic at neutral $\mathrm{pH}$ and desorbs principally by adding a proton to give a positive ion in the gas phase. The only metal-ion adducts for PC are monosodiated and monopotassiated for which a metal cation is added instead of $\mathrm{H}^{+}$. On the other hands, all other PL classes give mainly monosodiated as well as monopotassiated and disodiated species. For PA and PS, trisodiated species desorb owing to the presence of two acidic hydroxyl groups that make possible the addition of three sodium ions, two to replace acidic protons and one to charge the molecule [11]. Although the ionization patterns for PLs with ILMs as matrices are similar to those produced by 2,5-DHB [11], the protonated species of PE and PS, which are seen when 2,5-DHB is used, are not found with ILMs possibly because the ILM matrix is less acidic than 2,5-DHB.

The use of ILMs can lower the extent of prompt fragmentation of PLs. Others found [11] and we confirmed that prompt loss of the head group occurs for all PLs, except PC, when 2,5-DHB is used, but this loss is barely detectable with ILMs (Figure 1a versus Figure 1b). The major fragment for PC is the $\mathrm{m} / \mathrm{z} 184$ head-group ion. Two distinctive fragment ions of PC that form upon ESI [7] but were not reported for MALDI are of $\mathrm{m} / \mathrm{z} 496$ and 522 , arising by losses of ketene at sn-2 and sn-1, respectively. This occurs when an ILM or 2,5-DHB is used, but the extent is less with the ILM (data not shown).

In summary, ILMs have advantages over the crystalline 2,5-DHB matrix for MALDI analysis of PLs. Their use leads to higher signal intensity, comparable or better sensitivity, and improved sample homogeneity, leading to better signal reproducibility. Extension of ILM use to MS/MS and to on-line coupling of MALDI-MS to analytical and preparative LC [16] are future avenues for exploration, and implementation should be advantageous for the semi-quantitative and quantitative analysis of PLs in complex mixtures.

\section{Acknowledgments}

This research was supported by the National Centers of Research Resources of the National Institutes of Health of the U.S. (grant P41RR00954).

\section{References}

1. Pulfer, M.; Murphy, R. C. Electrospray mass spectrometry of phospholipids. Mass Spectrom. Rev. 2003, 22, 332-364.

2. Murphy, R. C.; Harrison, K. A. Fast-atom bombardment mass spectrometry of phospholipids. Mass Spectrom. Rev. 1994, 13, $57-75$.

3. Jensen, N. J.; Tomer, K. B.; Gross, M. L. Fast atom bombardment and tandem mass spectrometry of phosphatidylserine and phosphatidylcholine. Lipids 1986, 21, 580-588.

4. Han, X.; Gross, R. W. Electrospray ionization mass spectroscopic analysis of human erythrocyte plasma membrane phospholipids. Proc. Natl. Acsd. Sci. U.S.A. 1994, 91, 10635-10639.

5. Kim, H.-Y.; Wang, T.-C. L.; Ma, Y.-C. Liquid chromatography/mass spectrometry of phospholipids using electrospray ionization. Anal. Chem. 1994, 66, 3977-3982.

6. Hsu, F.-F.; Turk, J. Characterization of phosphatidylinositol, phosphatidylinositol-4-phosphate, and phosphatidylinositol4,5-bisphosphate by electrospray ionization tandem mass spectrometry: A mechanistic study. J. Am. Soc. Mass Spectrom. 2000, 11, 986-999.

7. Hsu, F.-F.; Turk, J. Electrospray ionization/tandem quadrupole mass spectrometric studies on phosphatidylcholines: The fragmentation processes. J. Am. Soc. Mass Spectrom. 2003, 14, 352-363.

8. Harvey, D. J. Matrix-assisted laser desorption/ionization mass spectrometry of phospholipids. J. Mass Spectrom. 1995, 30, 1333-1346.

9. Marto, J. A.; White, F. M.; Seldomridge, S.; Marshall, A. G. Structural characterization of phospholipids by matrix-assisted laser desorption/ionization Fourier transform ion cyclotron resonance mass spectrometry. Anal. Chem. 1995, 67, 3979-3984.

10. Schiller, J.; Arnhold, J.; Benard, S.; Muller, M.; Reichl, S.; Arnold, K. Lipid analysis by matrix-assisted laser desorption and ionization mass spectrometry: A methodological approach. Anal. Biochem. 1999, 267, 46-56.

11. Al-Saad, K. A.; Zabrouskov, V.; Siems, W. F.; Knowles, N. R.; Hannan, R. M.; Hill, H. H., Jr. Matrix-assisted laser desorption/ionization time-of-flight mass spectrometry of lipids: Ionization and prompt fragmentation patterns. Rapid Commun. Mass Spectrom. 2003, 17, 87-96.

12. Petkovic, M.; Schiller, J.; Muller, M.; Benard, S.; Reichl, S.; Arnold, K.; Arnhold, J. Detection of individual phospholipids in lipid mixtures by matrix-assisted laser desorption/ionization time-of-flight mass spectrometry: Phosphatidylcholine prevents the detection of further species. Anal. Biochem. 2001, 289, 202-216.

13. Armstrong, D. W.; Zhang, L.-K.; He, L.; Gross, M. L. Ionic liquids as matrixes for matrix-assisted laser desorption/ionization mass spectrometry. Anal. Chem. 2001, 73, 3679-3686.

14. Carda-Broch, S.; Berthod, A.; Armstrong, D. W. Ionic matrices for matrix-assisted laser desorption/ionization time-of-flight detection of DNA oligomers. Rapid Commun. Mass Spectrom. 2003, 17, 553-560.

15. Li, Y. L.; Gross, M. L. Ionic-liquid matrices for quantitative analysis by MALDI-TOF mass spectrometry. J. Am. Soc. Mass Spectrom. 2004, 14, 1833-1837.

16. Mank, M.; Stahl, B.; Boehm, G. 2,5-Dihydroxybenzoic acid butylamine and other ionic liquid matrixes for enhanced MALDI-MS analysis of biomolecules. Anal. Chem. 2004, 76, 2938-2950.

17. Estrada, R.; Yappert, M. C. Alternative approaches for the detection of various phospholipid classes by matrix-assisted laser desorption/ionization time-of-flight mass spectrometry. J. Mass Spectrom. 2004, 39, 412-422. 